ISSN: 2600-5859

\title{
Evaluación de la neuroeducación y su relación en el pro- greso de la enseñanza del aprendizaje del idioma Inglés
}

\section{Evaluation of neuroeducation and its relationship in the progress of teaching learning English language}

Vallejo Andrade Geovanna Elvira. ${ }^{1}$, Guambo Yerovi Enrique Jesús. ${ }^{2}$ \& Edison Renato Ruiz López. ${ }^{3}$

Recibido: 10-12-2019 / Revisado: 28-12-2019 /Aceptado: 04-01-2020/ Publicado: 07-02-2020

Abstract. DOI: $\quad$ https://doi.org/10.33262/concienciadigital.v3i1.1.1132

The objective of the research was to determine the relationship of neuroeducation in the process of teaching English language learning in students of the School of Nutrition and Diet of the Faculty of Public Health. For this, the dimensions of neuroeducation were identified and evaluated to know the components that define educational events. The research is qualitative-quantitative, descriptive, bibliographic, with an educational and field approach. The sample was intentional and was represented by 35 students of second "A" of the English level and 4 teachers. To obtain the results, two surveys at students and teachers were applied; They were reviewed by academic peers in the area and had a reliability of 0.8 according to Cronbach's alpha. The data were recorded, tabulated, analyzed and interpreted, and allowed to conclude that the neuroeducation used needs to be updated; because the surveys indicated that the learning developed is behavioral, although its objectives and contents were known by the students. However, the strategies used for the development of the activities require considering that the activities applied are motivational, dynamic and group; and consecuently in its programming they include technological tools to motivate autonomous and group work so that the process of learning English improves.

Keywords: Diagnosis, Neuroeducation, Teaching Learning, English

\footnotetext{
${ }^{1}$ Escuela Superior Politécnica de Chimborazo, Facultad de Salud Pública, Riobamba Ecuador, elvira.vallejo@espoch.edu.ec

${ }^{2}$ Escuela Superior Politécnica de Chimborazo, Centro de Idiomas, Riobamba - Ecuador, eguambo@espoch.edu.ec

${ }^{3}$ Escuela Superior Politécnica de Chimborazo, Ecuador, edisonr.ruiz@espoch.edu.ec
} 


\section{Resumen.}

El objetivo de la investigación fue determinar la relación de la neuroeducación en el proceso de enseñanza aprendizaje del idioma inglés en los estudiantes de la Escuela de Nutrición de la Facultad de Salud Pública. Para ello se identificó y evaluó las dimensiones de neuroeducación para conocer los componentes que definen los eventos educativos. La investigación es cuali-cuantitativa, de tipo descriptiva, bibliográfica, con enfoque educativo y de campo. La muestra fue intencional y estuvo representada por 35 estudiantes de cuarto "A" de la asignatura de inglés y 4 docentes. Para obtener los resultados se aplicaron dos encuestas dirigidas a estudiantes y docentes; las mismas que fueron revisada por pares académicos del área y que tuvo una confiabilidad de 0,8 según el alpha de Cronbach. Los datos fueron registrados, tabulados, analizados e interpretados, y permitieron concluir que la neuroeducación utilizada necesita debe ser actualizada; pues las encuestas indicaron que el aprendizaje desarrollado es conductista, a pesar de que sus objetivos y contenidos fueron conocidos por los estudiantes. Sin embargo, las estrategias utilizadas para el desarrollo de las actividades requieren considerar que las actividades aplicadas sean motivacionales, dinámicas y grupales; y que en su programación incluyan herramientas tecnológicas para motivar el trabajo autónomo y grupal con el fin de que el proceso del aprendizaje del idioma inglés mejore.

Palabras claves: Diagnóstico, Neuroeducación, Enseñanza aprendizaje, inglés

\section{Introducción.}

El diagnóstico mediante la identificación de para neuroeducación es base para que los docentes utilicen estrategias o enfoques de enseñanza que permita establecer una relación dinámica y cooperativa en el proceso de enseñanza y aprendizaje, para contribuir con la formación integral del estudiante. Por ello, es para el proceso de enseñanza aprendizaje se requiere que se diagnostique el actual enfoque y si amerita se aplique un nuevo enfoque de enseñanza, basado en el cerebro que permita crear y adquirir aprendizajes de una manera significativa, innovadora, dinámica y reflexiva, a través de la aplicación de estrategias neuroeducativas por parte de los docentes en el desarrollo de sus clases que faciliten y estimulen el desarrollo de las capacidades cognitivas, socio-emocionales y praxiológicas de los estudiantes para que el conocimiento impartido pueda ser empleado en el diario vivir. Según Aristizábal (2015), en su investigación, Avances de la neuroeducación y aportes en el proceso de enseñanza aprendizaje en la labor docente concluye que. "la neuroeducación que sin ser el método salvador si es una novedosa propuesta que desde la ciencia puede llegar a enriquecer el ejercicio profesional de los docentes, mediante la creatividad, la innovación y el conocimiento funcional cerebral del ser humano lograría transformar la práctica pedagógica" (p. 23) (Aristizábal, 2015, p. 23) 
González (2016) concluye que "la neuroeducación privilegia la enseñanza que respeta la libertad de las niñas y los niños como seres sociales que deben participar de la propia conducción de la enseñanza y que, por lo tanto, no hay razón científicamente valedera para imposiciones de cánones conductuales únicamente elaborados por adultos, con mucha información, pero que desconocen cómo es, cómo funciona y cómo aprende el cerebro de niños y niñas" (p. 423)

Según (Katzir y PareBlagoev, 2006) la neurociencia ha sido uno de los focos de atención en las últimas décadas, tanto en el área científica como en el área educativa. Los estudios en la neurociencia han ayudado a entender la relación de las estructuras del cerebro y sus funciones; mientras que Márquez (2002), sostiene que el docente debe conocer a los estudiantes desde el primer día de clases. Mediante un diagnostico e informe que establezca los aspectos relacionados al historial personal de cada estudiante, conductas frecuentes, reacciones extrañas, intereses y problemas más notables. Con el fin que el docente tenga una herramienta para incorporar la neurociencia en la sala de clases.

Gotay (2008) sostiene que en el proceso de aprendizaje es necesario valorar dos puntos fundamentales: el primero, el estado de ánimo del estudiante, es decir, la predisposición que éste tenga hacia la captación de una información nueva. Si el estudiante está contento, la información recibida será aprendida con mayor facilidad, de lo contrario, si el estudiante está desmotivado y está pasando por un problema emocional, las horas frente al educador no se aprovechan.

La Neuroeducación es una nueva visión de la enseñanza basada en el cerebro, que ha nacido al de la revolución cultural y que se ha denominado neurocultura. Por lo que neuroeducación es tomar ventaja de los conocimientos sobre cómo funciona el cerebro, en un intento de mejorar y potenciar tanto los procesos de aprendizaje, memoria de los estudiantes, y cómo enseñar mejor los profesores (Mora, 2013). Es así que este enfoque, da la oportunidad para orientar el aprendizaje en los procesos reflexivos del cerebro en busca de conocimientos significativos que contradicen a los modelos tradicionales.

Codina (2014) resume de manera muy clara y precisa los principios que guían la práctica educativa desde la perspectiva de la neuroeducación: Cada cerebro es único y únicamente organizado: es decir a pesar de que los humanos comparten patrones comunes de organización cerebral, ningún cerebro es igual a otro, puesto que todas las personas no piensan de la misma manera, pues el aprendizaje y el conocimiento depende mucho de la experiencia y del contexto social en el que se desenvuelva el individuo, considerando también cómo es adquirida la información.

El aprendizaje es percibido de modo diferente por cada persona; ya sea de manera kinestésica, auditiva o visual, por lo que el docente debe de comprender éste principio neuroeducativo 
para poder planificar diferentes actividades que favorezcan a todos los estilos de aprendizaje de los estudiantes, con el fin de potenciar la producción de aprendizaje significativo y duradero.

La editorial LISTIN DIARIO (2016), indica que al ser la Neuroeducación una ciencia naciente la información, las estrategias educativas son escasas; sin embargo, se expone las siguientes estrategias neuroeducativas: proyectos por equipos, el Juego, la educación física y el deporte, las emociones, las artes y la música y los Ipads y otros recursos tecnológicos. (LISTÍN DIARIO, 2016)

En base a lo indicado, la investigación plantea una línea base para que se tomen alternativas para la enseñanza aprendizaje del idioma inglés, a través del diagnóstico y análisis de los esquema conductual y tradicionales, a fin de establecer o proponer estrategia relacionadas con la neuroeducación. Las mismas que puedan ser utilizadas por los docentes en el desarrollo de las actividades en sus futras clases.

\section{Metodología.}

La investigación fue descriptiva, de campo y bibliográfica con enfoque cuali-cuantitativo, ya que es que estuvo relacionada con en el área de grado socioeducativa, a fin de conocer el propósito de la neuroeducación y cómo incide en el proceso de enseñanza aprendizaje del idioma inglés en los estudiantes de la escuela de Nutrición de la Facultad de Salud Pública. La presente investigación se apoyó desde un enfoque cuali-cuantitativo, ya que la información fue recolectada con un instrumento utilizando la escala de Likert a fin en cuantificar cualidades a valores numéricos de las variables del contexto de la investigación. La investigación tuvo carácter socioeducativo, pues se propuso una solución a un problema donde se intervino y se obtuvo la información de un conjunto de individuos desde el mismo (investigación de campo); además la información fue obtenida directamente de los intervenidos. Por otro lado la investigación es descriptiva ya que mediante la técnica de la encuesta y un instrumento (cuestionario) se recolecto los datos a fin de registrarlos, analizarlos e interpretarlos y permitieron establecer las respectivas conclusiones y recomendaciones de la investigación.

Con el fin de identificar la relación entre las variables independiente y dependiente de la investigación; se realizó la revisión bibliográfica con fuentes de primer y segundo orden de las variables Neuroeducación y el proceso de enseñanza aprendizaje del idioma inglés, es decir una investigación documental bibliográfica

La población por ser pequeña se utilizó su totalidad como una muestra intencional, la misma que está conformada por 35 estudiantes del nivel IV de inglés de la Carrera de Nutrición, Facultad de Salud Pública de la Escuela Superior Politécnica de Chimborazo ESPOCH, 
durante el período académico septiembre 2019 - febrero 2020. Los individuos tienen similares conocimientos, ya que aprobaron como requisito el nivel III de la asignatura de inglés basados en instrumentos pedagógicos (silabo, plan analítico, etc).

Tabla 1. Población y muestra

\begin{tabular}{ll}
\hline Muestra & $\mathbf{N}^{\circ}$ \\
\hline Docentes & 3 \\
Estudiantes & 35 \\
TOTAL & 38 \\
\hline
\end{tabular}

Fuente: Sistema Académico, ESPOCH

Elaborado por: Vallejo Geovanna, 2019

Para establecer los parámetros ha ser evaluados se consideró las siguientes dimensiones: Curiosidad, Respeto y saber escuchar

- Diferencias Individuales

- Interrelaciones sociales

- El juego

- Aprendizaje cooperativo

- Recursos tecnológicos

- Comunicativo (conversaciones y diálogos)

- Objetivos

\section{Contenidos}

Para la obtención de datos se utilizó la técnica de la encuesta y se elaboró dos cuestionarios que consideró la relación entre las variables. El primer cuestionario fue aplicado a los docentes; mientras que el segundo cuestionario fue aplicado a los estudiantes. El instrumento fue analizado, revisado y aprobado por cinco especialistas del área, y fue validado mediante el coeficiente Alfa de Cronbach, obteniendo un valor de confiablidad de 0,81; es decir fue bueno. (George y Mallery, 2003, p. 231)

La encuesta, permitió la finalidad en la obtención de los datos, a fin de dar respuesta a los objetivos propuestos, los cuestionarios contienen diez preguntas y tres alternativas de respuesta; Siempre, A Veces y Nunca. Para asegurar la coherencia con las variables, las dimensiones y las preguntas directrices, se elaboró los instrumentos en base a la matriz de operacionalización de variables.

Luego de la validación del instrumento, se procedió a su aplicación en la muestra seleccionada y los datos obtenidos fueron registrados, filtrados tabulados, analizados e interpretados, mediante tablas y gráficos de pastel; mediante la Microsoft Excel 
Para obtener la meta de la investigación se identificó principios de neuroeducación aplicados por los docentes que responden al desarrollo de procesos cognitivos del estudiante, luego se estableció el tipo de estrategia que utilizan los docentes en el desarrollo en su planificación curricular.

\section{Resultados}

\section{Encuesta a docentes}

Pregunta 1: ¿En el proceso del aprendizaje del idioma inglés, en el desarrollo de la curiosidad usa estrategias lúdicas?

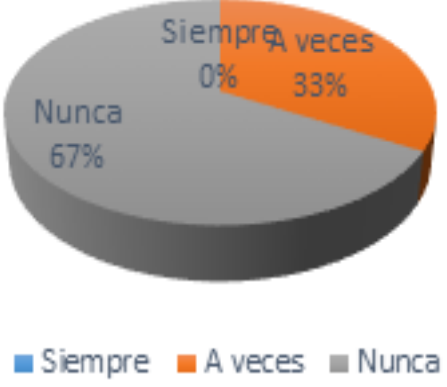

Gráfico 1. Uso de estrategias lúdicas en el desarrollo de la curiosidad

Del 100\% docentes encuestados, el $67 \%$ responde que Nunca usa alguna estrategia lúdica en el desarrollo de la curiosidad en el proceso de aprendizaje, el 33\% A veces; mientras que no registra frecuencia la opción Siempre. Es decir que si los docentes usan estrategias para motivar el desarrollo de la curiosidad, pero no lo hacen de forma continua, pues la mayoría responde que nunca. Por tanto es necesario que el personal docente presente en su plan de estudios el uso de estrategias, o a su vez se siga un modelo estándar que motive la dimensión del desarrollo de la curiosidad.

Pregunta 2: ¿Induce a desarrollar el proceso emocional mediante estrategias lúdicas?

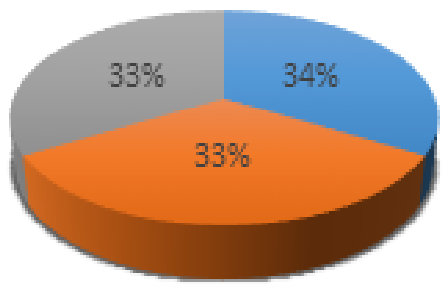

- Siempre A veces Nunca

Gráfico 2. Estrategias lúdicas en el desarrollo del proceso emocional 
Del $100 \%$ de docentes encuestados, el 34\% responde que Siempre Induce a desarrollar proceso emocionales mediante estrategias lúdicas, el 33\% A veces; mientras el $33 \%$ registra que la opción Nunca. Se debe considerar que solo un docente no aplica este tipo de estrategias que puede ocasionar problemas de respeto y escucha en el desarrollo de una clase.

Pregunta 3: ¿Para el proceso de enseñanza y aprendizaje, Usted planifica las actividades pedagógicas a fin de considerar las diferencias individuales?

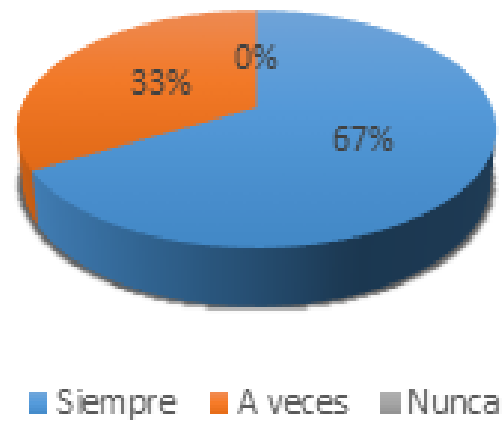

Gráfico 3. Actividades pedagógicas para considerar diferencias individuales

Del $100 \%$ de docentes encuestados, el $67 \%$ responde que Siempre planifica las actividades pedagógicas a fin de considerar las diferencias individuales en el proceso de enseñanza aprendizaje, el 33\% A veces; mientras que no registra frecuencias la opción Nunca. Es decir que los docentes proveen las diferencias individuales mediante las actividades contempladas en sus planes analíticos, incluyendo o abracando las diferencias de aprendizajes del idioma inglés. Es decir, los docentes preparan correctamente sus actividades pedagógicas.

Pregunta 4: ¿En el proceso de enseñanza y aprendizaje del idioma inglés, usted motiva el desarrollo del cerebro con interrelaciones sociales?

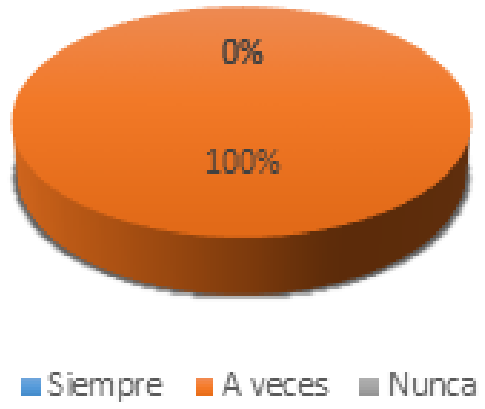

Gráfico 5. Planificación de actividades colaborativas para aprendizajes neuroeducativos 
ISSN: 2600-5859

Vol. 3, $\mathrm{N}^{\circ} 1.1$, p. 65-77, febrero, 2020

Del $100 \%$ de docentes encuestados, el $67 \%$ responde que $A$ veces planifica actividades colaborativas para aprendizajes neuroeducativos; mientras el 33\% la opción Nunca. Por lo que, no se hace énfasis en el uso de actividades colaborativas que son necesarias para que el aprendizaje tenga sus efectos.

Pregunta 6. ¿Expone a sus estudiantes el objetivo y contenido de cada unidad a desarrollar?

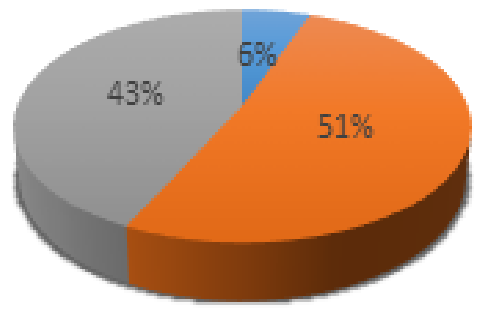

- Siempre a veces $n$ Nunca

Gráfico 6. Exposición de objetivo y contenido de unidades a desarrollar

Del $100 \%$ de docentes encuestados, el $67 \%$ responde que Siempre expone los objetivo y contenido de unidades a desarrollar; mientras el 33\% la opción A veces. Es decir que la mayoría de los docentes comparten con los estudiantes la meta y los temas que se van a desarrollar en las unidades; por lo que los estudiantes conocen el camino a seguir.

Pregunta 7: ¿Utiliza el juego como estrategia neuroeducativa, para el en el desarrollo cognitivo?

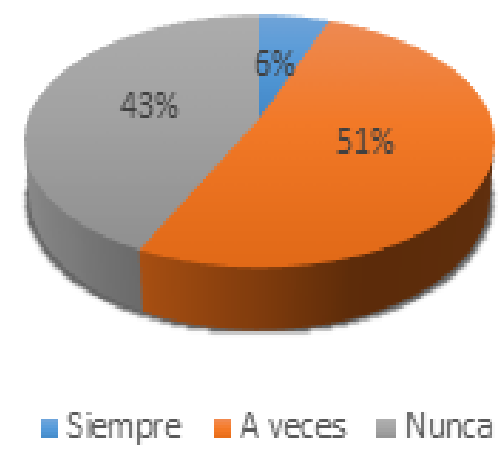

Gráfico 7. El juego como estrategia neuroeducativa, para el en el desarrollo cognitivo

Del $100 \%$ de docentes encuestados, el $67 \%$ responde que Nunca utiliza el juego como estrategia neuroeducativa, para el en el desarrollo cognitivo; mientras el 33\% la opción $A$ 
ISSN: 2600-5859

Vol. 3, $\mathrm{N}^{\circ} 1.1$, p. 65-77, febrero, 2020

veces. Es decir que la mayoría de los docentes consideran que no es necesario estrategias donde el juego sea un factor de interactividad para que se utilicen como forma de desarrollo cognitivo, ignorando que son métodos que implican alternativas para el proceso de aprendizaje.

\section{Encuesta a estudiantes}

Pregunta 1: ¿Al realizar actividades lúdicas en inglés, usted experimenta curiosidad?

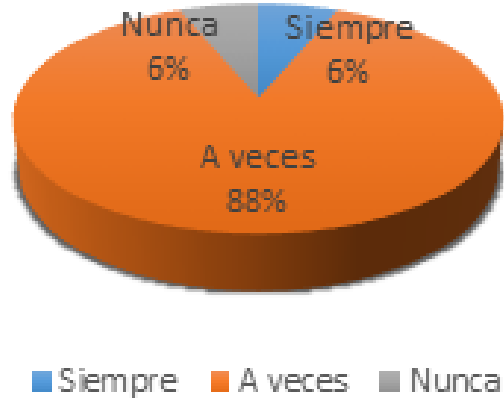

Gráfico 8. Curiosidad al realizar actividades lúdicas en inglés

Del total de los 35 estudiantes consultados, el $88 \%$ responde que $A$ veces experimenta curiosidad al realizar actividades lúdicas en inglés; el 6\% indica que Siempre; mientras el 6\% responde que Nunca. Por lo que la mayoría de los estudiantes desarrollan curiosidad cuando realizan actividades lúdicas; es decir que se debe hacer énfasis para que la frecuencia la opción $A$ veces mejore.

Pregunta 2: ¿Durante las actividades recreativas de las clases de inglés, usted sigue las instrucciones indicadas por el docente?

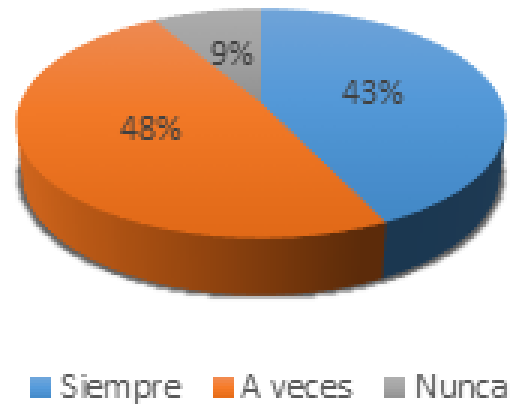

Gráfico 9. Cumplimiento de instrucciones del docente en actividades recreativas. Del $100 \%$ de estudiantes consultados, el $48 \%$ responde que $A$ veces cumplen con las instrucciones del docente en actividades recreativas; el $48 \%$ indica que Siempre; mientras el 
9\% responde que Nunca. Es decir que pocos estudiantes están desatendiendo las instrucciones que deben ser cumplidas con el objetivo de inducir a un correcto aprendizaje.

Pregunta 3: ¿En el desarrollo de las clases, usted actúa de acuerdo a su propio ritmo en el aprendizaje del inglés?

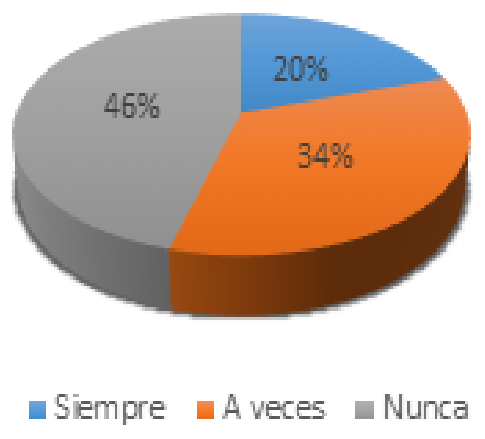

Gráfico 10. Ritmo de aprendizaje en el desarrollo de clases de inglés.

Del $100 \%$ de estudiantes encuestados, el $46 \%$ responde que Nunca siguen su propio ritmo de aprendizaje en el desarrollo de clases de inglés; el 34\% indica que A veces; mientras el $20 \%$ responde que Siempre. Es decir que las clases están siendo conductistas, que el plan de clase es muy claro, o por puede acreditarse a que el grupo es heterogéneos en aprendizaje.

Pregunta 4: ¿Al relacionarse con sus compañeros estudiantes, puede expresarse con facilidad en la clase de inglés?

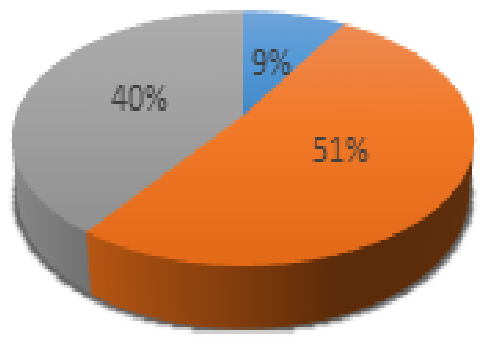

-Siempre $\square$ A veces $n$ Nunca

Gráfico 11. Facilidad para relacionarse cuando se expresan.

Del $100 \%$ de estudiantes encuestados, el $51 \%$ responde que $A$ veces puede expresarse con facilidad para relacionarse con sus compañeros en la clase de inglés; el $40 \%$ indica que 
ISSN: 2600-5859

Vol. 3, $\mathrm{N}^{\circ} 1.1$, p. 65-77, febrero, 2020

Nunca; mientras el $9 \%$ responde que Siempre. Por lo que la mayoría de los estudiantes presentan problemas para relacionarse; es decir, que debe mejorar su lenguaje y sus expresiones e interactividad.

Pregunta 6: ¿Durante las clases, participa en actividades colaborativas o grupales indicadas por el docente?

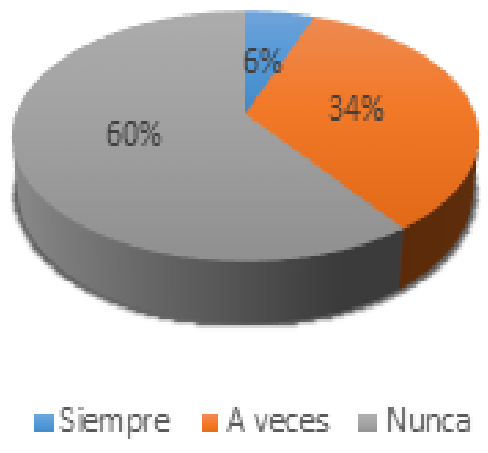

Gráfico 12. Facilidad para relacionarse cuando se expresan.

Del $100 \%$ de estudiantes encuestados, el $60 \%$ responde que Nunca participa en actividades colaborativas o grupales indicadas por el docente; el 34\% indica que A veces; mientras el $6 \%$ responde que Siempre. Es decir que el trabajo colaborativo está siendo desplazado por actividades conductitas, por lo que no se construye el conocimiento de forma grupal.

Pregunta 6: ¿En las clases de inglés, participa en actividades que involucran juegos?

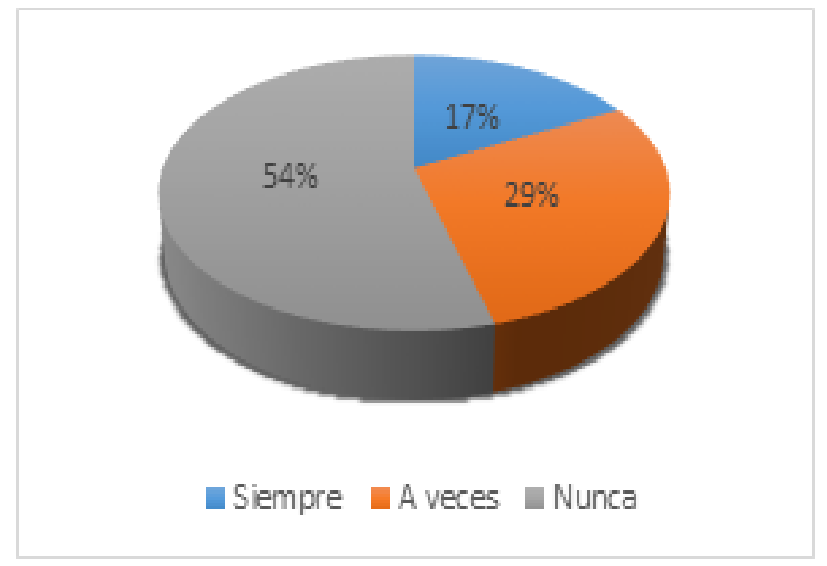

Gráfico 13. Participación en actividades que involucran juegos 
Del 100\% de estudiantes encuestados, el 54\% responde que Nunca participan en actividades que involucran juegos; el $29 \%$ indica que A veces; mientras el $17 \%$ responde que Siempre. Es decir, que la mayoría de los estudiantes participan sin motivación que puede interpretarse por la falta de liderazgo, desorganización de la actividad o desinterés.

Pregunta 7: ¿Para reforzar el aprendizaje del idioma inglés, utiliza recursos tecnológicos?

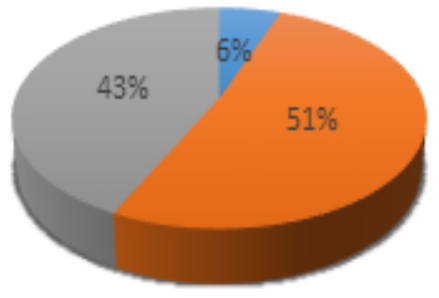

- Siempre A veces nunca

Gráfico 14. Uso de recursos tecnológicos para reforzar el aprendizaje

Del $100 \%$ de estudiantes encuestados, el $51 \%$ responde que $A$ veces utiliza recursos tecnológicos para reforzar el aprendizaje del idioma ingles; el 43\% indica que Nunca; mientras el 6\% responde que Siempre. Es decir, que posiblemente no se están involucrando las TIC en las actividades programadas para que los estudiantes de forma autónoma, utilicen herramientas tecnológicas para reforzar el aprendizaje del idioma inglés. Por lo que es necesario actualizar los planes analíticos de la asignatura del idioma inglés.

Pregunta 8: ¿Conoce los objetivos y contenidos de cada unidad de la asignatura de inglés?

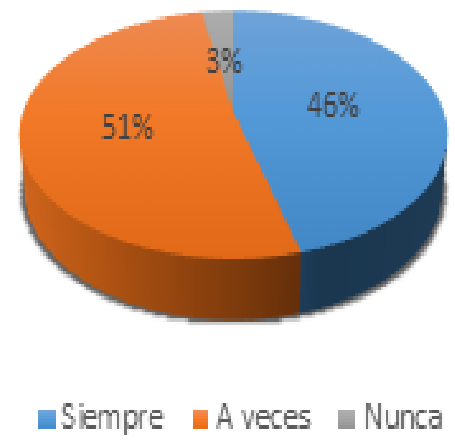

Gráfico 15. Conocimiento de objetivos y contenidos de cada unidad de la asignatura de inglés 
Del 100\% de estudiantes encuestados, el 51\% responde que $A$ veces conoce los objetivos y contenidos de cada unidad de la asignatura de inglés; el $46 \%$ indica que Nunca; mientras el $3 \%$ responde que Nunca. Es decir que la mayoría de los estudiantes tienen conocimiento del camino para conseguir el aprendizaje del idioma ingles por cada unidad para su aprendizaje.

\section{Conclusiones.}

- Se identificó que los principios de neuroeducación aplicados por los docentes en el proceso de enseñanza y aprendizaje del idioma inglés en el cuarto nivel de idioma inglés, responden al desarrollo de procesos cognitivos del estudiante; ya que los docentes planifican actividades grupales, sin embrago deben ser fortalecidas y dirigidas de manera más efectiva ya que el interés y desmotivación de los estudiantes se reflejan en los porcentajes obtenidos de las encuestas. Es decir, que los principios que responden a procesos emocionales son utilizados con poca frecuencia.

- Se estableció que las estrategias neuroeducativas que emplean los docentes del idioma inglés, entre ellas el recurso tecnológico deben ser contemplados y fortalecidos en su planificación de estudios o planes de clase, con el fin de que su uso, se refleje en la incidencia del aprendizaje. A la vez, pueden ser utilizados en retroalimentación y evaluación que deberán ser enunciado en las rúbricas para que el estudiante se motive y presente interés. Pues una educación que no se adapta a la actualidad, es una educación condenada a no cumplir con sus objetivos.

- Por su parte los docentes socializan con sus estudiantes los contenidos y objetivos de los temas a ser estudiados durante las clases del idioma inglés, mismos que son componentes indispensables del proceso de enseñanza y aprendizaje; sin embargo, las estrategias que están siendo aplicadas necesitan enfocarse en las teorías constructivistas, ya se nota que se están incurriendo en el conductismo.

\section{Referencias Bibliográficas.}

Aristizábal, A. (2015). avances de la neuroeducación y aportes en el proceso de enseñanza aprendizaje en la labor docente [Tesis, Universidad Militar Nueva Granada]. https://repository.unimilitar.edu.co/bitstream/handle/10654/6186/Trabajo\%20Final. pdf;jsessionid=2AB9C3D0A771810A3D0915D169F7E213?sequence $=1$

Codina, M. J. (2014). Neuroeducación en virtudes cordiales: una propuesta a partir de la neuroeducación y la ética discursiva cordial. [Tesis de doctorado]. Universidad de Valencia, Valencia. Recuperado el 26 de junio de 2016, de http://roderic.uv.es/bitstream/handle/10550/35898/TesisCodinaMJ.pdf?sequence=1 \&isAllowed $=\mathrm{y}$

Katzir, T. y ParéBlagoev, J. (2006). Applying cognitive neuroscience, research to education: The case of literacy. Educational Psychologist.41, 1

George, D., \& Mallery, P. (2003). SPSS for Windows step by step: A simple guide and reference. 11.0 update (4thed.). Boston: Allyn \& Bacon 
ISSN: 2600-5859

Vol. 3, $\mathrm{N}^{\circ} 1.1$, p. 65-77, febrero, 2020

González, C. (2016). Neuroeducación y lingüística: Una propuesta de aplicación a la enseñanza de la lengua materna [Doctoral, Universidad Complutense De Madrid]. https://eprints.ucm.es/35929/1/T36890.pdf

Gotay, Y. (2008). Neurociencia: Herramienta para facilitar el aprendizaje [Universidad Metropolitana].

https://dheducacion.com/wpcontent/uploads/2019/02/Neurociencia-Herramienta-Para-Facilitar-El-

Aprendizaje.pdf

Listín Diario. (2016). Estrategias de neuroeducación [Pagina LEA]. https://planlea.listindiario.com/2016/05/estrategias-de-neuroeducacion/

Márquez, H. (2002). Aprendiendo a expresar emociones. Puerto Rico: Camera Mundi, Inc.

Mora, F. (2013). Neuroeducación: solo se puede aprender aquello que se ama. Madrid, España: Alianza Editorial S. A. Recuperado el 23 de junio de 2016, de https://es.scribd.com/doc/305882367/Neuroeducacion-de-Francisco-Mora 
ISSN: 2600-5859

PARA CITAR EL ARTÍCULO INDEXADO.

Geovanna Elvira, V. A., Enrique Jesús, G. Y., \& Ruiz López, E. R. (2020). Evaluación de la neuroeducación y su relación en el pro-greso de la enseñanza del aprendizaje del idioma Inglés. ConcienciaDigital, 3(1.1), 65-77. https://doi.org/10.33262/concienciadigital.v3i1.1.1132

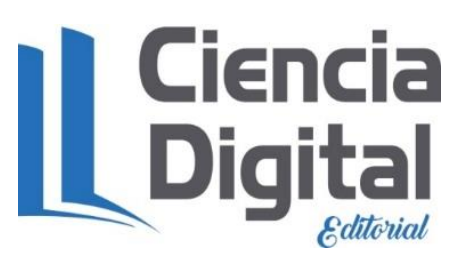

El artículo que se publica es de exclusiva responsabilidad de los autores y no necesariamente reflejan el pensamiento de la Revista Conciencia Digital.

El artículo queda en propiedad de la revista y, por tanto, su publicación parcial y/o total en otro medio tiene que ser autorizado por el director de la Revista Conciencia Digital.
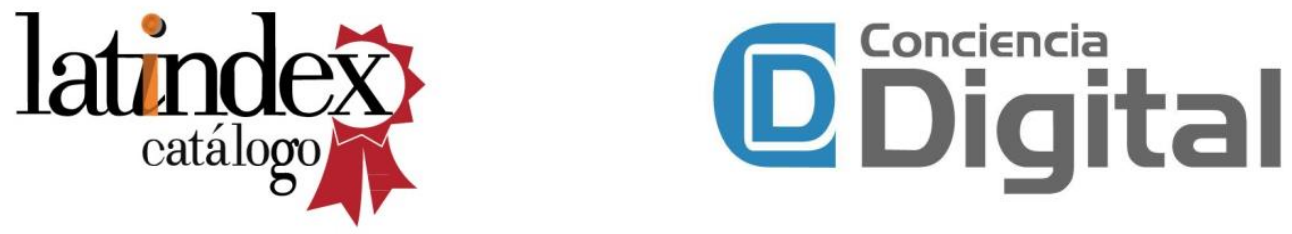